\title{
Personal view
}

\section{Congenital dislocation of the hip: to screen or not to screen}

\author{
N StJ P DWYER
}

East Birmingham Hospital, Birmingham

\section{History of screening}

Detection of congenital dislocation of the hip by clinical examination in newborn infants was described in 1937 by Ortolani. ${ }^{1}$ The routine use of such an examination in all newborn babies, plus the beginning of immediate conservative management in all detected cases, was reported in 1952 by Von Rosen. ${ }^{2}$ Von Rosen's approach promised an end to the missed late cases so often requiring multiple, hazardous, and incompletely successful surgical procedures. Indeed, in the wave of enthusiastic optimism, with which his programme was taken up in most major centres, it was expected that congenital hip dysplasia would become a condition always detected in the neonatal period and successfully treated by simple splintage within the first few months of infancy. Unfortunately, this did not prove to be the case, and although most screens provided an improvement in diagnostic success, many centres outside Sweden, continued to report undiagnosed cases. This experience was reported by Barlow in 1962 when he described a supplementary clinical test to add to the Ortolani manoeuvre. ${ }^{3}$

There is no doubt that Barlow's test would certainly detect unstable hips that might be missed using only the Ortolani manoeuvre, and both these tests have remained the basis of clinical screening for congenital dislocation of the hip. Cases continue, however, to slip through the meshes of the diagnostic screen. Furthermore, again in centres outside Sweden, cases of proximal capital epiphysitis began to be reported. This epiphysitis is the development of a Perthes like lesion in the proximal capital femoral epiphysis and is the result of splintage in abduction. ${ }^{4}$ This is a serious complication and one that is heightened by the knowledge that many of the unstable or dislocated hips detected at birth will relocate and stabilise spontaneously without any treatment.

\section{Timing of the screen}

There have been two quite different responses to these problems among those with a special interest in congenital hip dysplasia. One response has been to attempt to make neonatal screening more successful and primary treatment by splintage safer. The other response has been to abandon neonatal screening and splintage and start a screen of all infants at 3 months of age.

I would like first to examine the second response. The overriding advantage of this approach is that those cases that are to resolve spontaneously will have done so by 3 months, and these normal babies will not have been exposed to unnecessary and potentially hazardous treatment. Furthermore, it is generally easier to diagnose a dislocated hip at 3 months. It must be said that this is an expression, however, of the development of adaptive changes, and thus deterioration of the deformity, which could well be avoided by prompt successful neonatal splintage.

There are other problems with this approach. The results of treatment at 3 months are not as good and are probably going to require surgical as opposed to conservative management. Indeed, some of us believe that congenital dislocation of the hip that cannot be treated conservatively in the neonatal period is best managed by soft tissue release and open relocation of the hip before the onset of walking. ${ }^{5}$ Certainly, satisfactory management in purely conservative terms is unlikely to be adequate when treatment is started as late as 3 months of age, and if it is attempted the chances of epiphysitis occurring are certainly much higher than when a child is loosely splinted in the early perinatal period.

The first response, aimed at improving the efficiency of a neonatal screen and the safety of splinting, has, I believe, the best chances of overall success in the management of hip dysplasia. There are two ways in which the screen can be improved. 
The first involves improvement in expertise required to carry out efficiently the clinical examination. The second involves increasing the amount of information from which a diagnosis might be made.

Improvement of expertise. The actual business of examining babies' hips at birth is often left to relatively inexperienced junior staff. With a few hours of intensive instruction from an experienced teacher, it is probably possible to make such young doctors reasonably efficient in that they will be able to pick out the obvious dislocated or dislocatable hip.

Over the past nine years we have examined the incidence of congenital dislocation of the hip occurring in the newborn population of a large maternity unit with roughly 3000 live births each year. During the first two years it was obvious that some positive misdiagnoses were being made, but after the first two years the incidence fell to a steady rate of just under $1 \%$ of live births. This suggested very strongly to us that two years' experience were required to avoid making false positive diagnoses.

A similar but longer learning curve was noted in the number of cases diagnosed after the neonatal period. The first year of the study showed the number of cases diagnosed was 1.6 per 1000 live births, falling to 0.33 five years later. During the period 1983-85 there were no late diagnosed cases and in 1986 there has been one. This would suggest that our team's technique continued to improve over a period of four years. All but two of these late cases were in the high risk group, which was followed up routinely at 3 months, even though they had been found to be apparently normal at birth. These results were achieved by a team of three people that has remained the same throughout the survey. The team consists of one orthopaedic surgeon and two senior physiotherapists, who screen every live birth. ${ }^{6}$

Improvement of information. At present in most centres the basis of information is purely clinical and depends on interpretation of the feel of the hips on clinical examination. The very obviously unstable clunking hip is easy, but even with considerable experience the differentiation of a low pitched click from a minimal clunk can be difficult. Detection of the sliding hip, which gives a minimal sensation as it slides across the lip of the acetabulum in a lax joint, can require a high degree of clinical acumen earned by years of experience and constant practice.

Two new exciting aids to diagnosis are being developed. The first, which involves ultrasound screening of the neonatal hip, is very promising. ${ }^{7}$ Unlike $x$-ray films, ultrasound outlines the soft tissue and is rapidly becoming more reliable as the expertise of the operators increases and the efficiency of the machines improves. The second interesting experimental approach is that of Professor Mollen from Belfast, who is using electronic visual and auditory display to give a more objective appreciation for the Barlow and Ortolani tests.

\section{Safety of splintage for congenital dislocation of the hip}

Since 1977 we have not had one case of epiphysitis as a result of Von Rosen splintage, with an average birth rate of 3000 each year and an incidence of congenital dislocation of the hip of just under $1 \%$. How is this achieved?

I believe that epiphysitis only occurs if the femoral head is jammed rigidly into the acetabulum by forcibly abducting the joint against a tight medial joint capsule. We therefore apply the Von Rosen splint in a manner that allows free movement in abduction. Only very rarely in the perinatal period is the capsule so tight that reduction is difficult, and in these rare cases the splint is not applied, the hip being left free for review at 3 months. The structures around the hip rapidly tighten up and we therefore apply the splint at 72 hours. This allows a small proportion of hips to relocate spontaneously while maintaining easy reduction without force in the remainder.

\section{The screen}

The screen that we use in my unit is similar in many respects to many others in Britain.

On day 1 each baby undergoes the Barlow and Ortolani examination of the hips. The following situations may arise.

(1) If the hips are found to be normal and the child does not fall into a high risk group the patient is discharged from follow up.

(2) If they are apparently normal but in a high risk group (breech presentation, moulding defect, or family history of congenital dislocation of the hip) they are re-examined at 3 months.

(3) If they have one or both hips dislocatable yet freely relocatable they are re-examined on the next two days. If during this time there is no change they are loosely splinted in abduction for three months and then re-examined.

(4) If during these three days the hip or hips stabilise then they are not splinted but reexamined at 3 months.

(5) Rarely, one or both hips may be found to be rigidly dislocated and not easily relocatable. 
In these cases splinting is not attempted and the baby is re-examined at 3 months.

Groups 2 to 5 undergo examination at 3 months. If there is a doubt or distinct suspicion of dislocation or subluxation the hips are examined under general anaesthetic and an arthrogram is carried out. If the clinical examination is apparently normal the babies are examined again at 7 months and have a single plain anteroposterior $x$-ray film taken of the pelvis with the hips in neutral position. If this $x$-ray and clinical examination yield normal results the child is checked once again at 14 months for assessment of gait. If the $x$-ray film shows a dislocation or suggests a subluxation then an arthrogram and a clinical examination are carried out under general anaesthetic. If the arthrogram carried out at 3 or 7 months shows a dislocated or subluxed hip the infant undergoes an open reduction of the hip. All these children are followed up until growth has ceased. If the arthrogram is normal when carried out at 3 months a plain $x$-ray film is taken at 7 months.

We believe that this programme could theoretically be improved by adding the apparently normal babies (group 1) into the 3 month follow up. This would mean screening all babies in both the neonatal and three monthly periods. In our experience in over nine years and 28000 births two babies with dislocated hips slipped through our screen, having been excluded from the 3 month follow up because they were apparently normal at birth and did not fall into any of the high risk groups.

\section{Conclusion}

It is possible to achieve a neonatal screening programme for congenital dysplasia of the hip that is efficient in terms of making an accurate diagnosis. It requires a dedicated, experienced, and skilful team, however, to achieve this. I have no doubt that the efficiency of the screening process can be further improved by use of the newer diagnostic aids, such as electronic augmentation for interpretation of the clinical signs and ultrasound scanning of neonatal hips. Equally, I am quite sure that these aids will not compensate for lack of skill or training in those responsible for the screen.

We have shown that with care and strict attention to timing it is possible to begin primary conservative splintage of the dislocated hip with complete safety. We feel that this really removes the main advantage gained by abandoning the neonatal screen for one carried out at 3 months of age and furthermore, dramatically improves the expectations of success.

\section{References}

1 Ortolani M. Un Segno poco noto e sua importanza per la diagnosi precoce di prelussazione congenita dell'anca. Pediatria (Napoli) 1937:45:129-36.

2 Von Rosen S. Diagnosis and treatment of congenital dislocation of the hip in the new-born. J Bone Joint Surg [Br] 1962;44: 284-91.

3 Barlow TG. Early diagnosis and treatment of congenital dislocation of the hip. J Bone Joint Surg /Br] 1962;44:292-301.

4 Wilkinson JA. Failures in the management of congenital hip displacement in the new-born. Proceedings of the Royal Society of Medicine 1975:68:476-9.

5 O'Hara J, Bernard AA, Dwyer NStJ. Medial approach open reduction in congenital dislocation of the hip before walking age. J Bone Joint Surg $\mid \mathrm{Br} /$.

' Bernard AA, O'Hara J, Dwyer NStJ. An improved screening system for the early detection of congenital dislocation of the hip (in a peripheral hospital). J Pediatr Orthop. (In press.)

${ }^{7}$ Clarke NPM, Marcke HT, McHugh P, Lee MYS, Borns PF, MacEwen GD. Real-time ultrasound in the diagnosis of congenital dislocation and dysplasia of the hip. J Bone Joint Surg [Br] 1985;87:406-22.

Correspondence to Mr N StJ P Dwyer, East Birmingham Hospital, Bordesley Green East, Birmingham B9 5ST, England. 